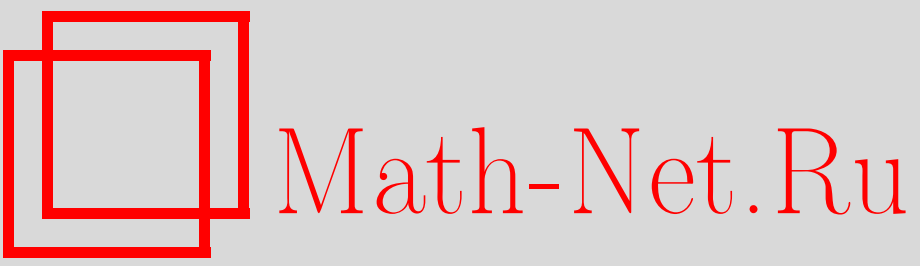

В. Г. Задорожний, О нахождении моментных функций решения задачи Коши уравнения диффузии со случайными коэффициентами, Изв. РАН. Сер. матем., 2002, том 66, выпуск 4, 119-136

DOI: https://doi.org/10.4213/im396

Использование Общероссийского математического портала Math-Net.Ru подразумевает, что вы прочитали и согласны с пользовательским соглашением

http://www. mathnet.ru/rus/agreement

Параметры загрузки:

IP : 54.198 .64 .247

26 апреля 2023 г., 10:30:13 


\section{О нахождении моментных функций решения задачи Коши уравнения диффузии со случайными коэффициентами}

\footnotetext{
Рассматривается задача Коши для дифференциального уравнения диффузии одной пространственной переменной. Коэффициенты уравнения и начальные условия являются случайными процессами. Получены формулы для первой и второй моментных функций решения.

Библиография: 16 наименований.
}

1. Постановка задачи. Пусть $\mathbb{R}$ - вешественная ось, $\mathbb{C}$ - комплексная плоскость, $\left[t_{0}, t_{1}\right]=T \in \mathbb{R}, L_{1}(T)$ - пространство суммируемых на отрезке $T$ функций, $L_{\infty}(T)-$ пространство существенно ограниченных на $T$ функций $[1], F_{x}[f](\xi)$ - преобразование Фурье [2] по переменной $x, F_{\xi}^{-1}$ - обратное преобразование Фурье по переменной $\xi$, знак * обозначает свертку функций по переменной $x$.

Пусть $X$ - банахово пространство функций на отрезке $T$ и $y: X \rightarrow \mathbb{C}$. Если дифференциал $\Phi$ реше [3, c. 469] $d y\left(x_{0}, h\right)$ этого функционала в точке $x_{0}$ имеет вид

$$
d y\left(x_{0}, h\right)=\int_{T} \varphi\left(t, x_{0}\right) h(t) d t
$$

где интеграл понимается в смысле Лебега, $\varphi: T \times X \rightarrow \mathbb{C}$, то $\varphi\left(t, x_{0}\right)$ называется вариачионной (функииональной) производной (см. [4], [5]) функционала $y$ в точке $x_{0}$ и обозначается $\delta y\left(x_{0}\right) / \delta x(t)$.

Задача Коши для уравнения диффузии имеет вид

$$
\begin{gathered}
\frac{\partial u(t, x)}{\partial t}=\varepsilon_{1}(t) \frac{\partial u(t, x)}{\partial x}+\varepsilon_{2}(t) \frac{\partial^{2} u(t, x)}{\partial x^{2}}+f(t, x), \\
u\left(t_{0}, x\right)=u_{0},
\end{gathered}
$$

где $u$ - искомая функция, $\varepsilon_{1}: T \rightarrow \mathbb{R}, \varepsilon_{2}: T \rightarrow \mathbb{R}, f: T \times \mathbb{R} \rightarrow \mathbb{R}, u_{0}: \mathbb{R} \rightarrow \mathbb{R}$ - случайные процессы (зависимость от случайного события в записи не отражается). Предполагается, что случайный процесс $u_{0}$ не зависит от случайных процессов $\varepsilon_{1}, \varepsilon_{2}, f$.

Будем предполагать, что случайные процессы $\varepsilon_{1}, \varepsilon_{2}, f$ заданы характеристическим функционалом, т. е. известно

$$
\varphi\left(v_{1}, v_{2}, w\right)=\operatorname{Me}\left(v_{1}, v_{2}, w\right)
$$


где $\mathrm{M}$ - знак математического ожидания по функции распределения процессов $\varepsilon_{1}, \varepsilon_{2}, f$ и

$$
\begin{aligned}
e\left(v_{1}, v_{2}, w\right)= & \exp \left(i \int_{T}\left[\varepsilon_{1}(s) v_{1}(s)+\varepsilon_{2}(s) v_{2}(s)\right] d s\right. \\
& \left.+i \int_{T} \int_{\mathbb{R}} f\left(s_{1}, s_{2}\right) w\left(s_{1}, s_{2}\right) d s_{1} d s_{2}\right)
\end{aligned}
$$

$v_{1}, v_{2} \in L_{1}(T), w \in L_{1}(T \times \mathbb{R})$.

Требуется найти моментные функции решения задачи $(1),(2)$.

Для нахождения моментных функций решений дифференциальных уравнений, коэффициенты которых являются случайными процессами, применяется несколько подходов. Строятся цепочки дифференциальных уравнений для моментных функций. Как правило, такие цепочки бывают связанными, бесконечными и незамкнутыми. Иногда их удается разорвать или замкнуть [6]-[8].

Для некоторых задач решение можно выписать в явном виде через коэффициенты уравнения и затем найти моментные функции. Таким способом получены первые две моментные функции решения задачи Коши для линейного обыкновенного дифференциального уравнения второго порядка с гауссовскими коэффициентами [9], [10].

Для уравнений с малыми случайными возмущениями строятся асимптотические приближения [11]. Развита теория стохастических дифференциальных уравнений.

В случае линейных дифференциальных уравнений задача может быть сведена к дифференциальным уравнениям с вариационными производными (см. [12], [4], [5]). Этим способом удалось получить формулы для моментных функций решений линейных дифференциальных уравнений в частных производных первого порядка [13] и для дифференциального уравнения теплопроводности (см. [4], [14], [15]). В настоящей работе получена рекуррентная последовательность дифференциальных уравнений с вариационными производными, из решений которой легко находятся моментные функции. Для первых двух моментных функций получены явные формулы.

2. Уравнение для характеристического функционала. Введем обозначения

$$
\begin{gathered}
e_{1}\left(v_{1}, v_{2}, w, z\right)=\exp \left(i \int_{T}\left[\varepsilon_{1}(s) v_{1}(s)+\varepsilon_{2}(s) v_{2}(s)\right] d s\right. \\
\left.+i \int_{T} \int_{\mathbb{R}}\left[f\left(s_{1}, s_{2}\right) w\left(s_{1}, s_{2}\right)+u\left(s_{1}, s_{2}\right) z\left(s_{1}, s_{2}\right)\right] d s_{1} d s_{2}\right), \\
Y\left(v_{1}, v_{2}, w, z\right)=\operatorname{M} e_{1}\left(v_{1}, v_{2}, w, z\right),
\end{gathered}
$$

где $z \in L_{1}(T \times \mathbb{R}), M$ - знак математического ожидания по функции распределения процессов $\varepsilon_{1}, \varepsilon_{2}, f, u_{0}$.

Умножим уравнение $(1)$ на $e_{1}\left(v_{1}, v_{2}, w, z\right)$. При этом математическое ожидание полученного равенства формально можно записать с помошью отображения $Y$ в виде

$$
\frac{1}{i} \frac{\partial}{\partial t} \frac{\delta Y}{\delta z(t, x)}=\frac{1}{i^{2}} \frac{\delta}{\delta v_{1}(t)} \frac{\partial}{\partial x} \frac{\delta Y}{\delta z(t, x)}+\frac{1}{i^{2}} \frac{\delta}{\delta v_{2}(t)} \frac{\partial^{2}}{\partial x^{2}} \frac{\delta Y}{\delta z(t, x)}+\frac{1}{i} \frac{\delta Y}{\delta w(t, x)}
$$


или

$$
\frac{\partial}{\partial t} \frac{\delta Y}{\delta z(t, x)}=-i \frac{\delta}{\delta v_{1}(t)} \frac{\partial}{\partial x} \frac{\delta Y}{\delta z(t, x)}-i \frac{\delta}{\delta v_{2}(t)} \frac{\partial^{2}}{\partial x^{2}} \frac{\delta Y}{\delta z(t, x)}+\frac{\delta Y}{\delta w(t, x)}
$$

Умножая (2) на $e_{1}\left(v_{1}, v_{2}, w, z\right)$ и переходя к средним значениям, получаем

$$
\left.\frac{\delta Y\left(v_{1}, v_{2}, w, z\right)}{\delta w(t, x)}\right|_{t=t_{0}}=i \mathrm{M}\left(u_{0}(x) e_{1}\left(v_{1}, v_{2}, w, z\right)\right)
$$

Таким образом, для $Y$ получена детерминированная задача (3), (4), причем коэффициенты уравнения (3) не зависят от статистических характеристик процессов $\varepsilon_{1}, \varepsilon_{2}, f$, $u_{0}$. Если известно $Y$, то легко находятся моментные функции решения задачи $(1),(2)$ и даже корреляционные функции процессов $\varepsilon_{1}, \varepsilon_{2}, f, u$. Например,

$$
\begin{aligned}
\mathrm{M} u(t, x) & =\left.\frac{1}{i} \frac{\delta Y\left(v_{1}, v_{2}, w, z\right)}{\delta z(t, x)}\right|_{v_{1}=0, v_{2}=0, w=0, z=0} \\
\mathrm{M}\left(u(t, x) \varepsilon_{1}(s)\right) & =-\left.\frac{\delta^{2} Y\left(v_{1}, v_{2}, w, z\right)}{\delta z(t, x) \delta v_{1}(s)}\right|_{v_{1}=0, v_{2}=0, w=0, z=0}
\end{aligned}
$$

Таким образом, важно найти решение задачи $(3),(4)$ в малой окрестности точки $(0,0,0,0)$ переменных $v_{1}, v_{2}, w, z$.

3. Степенной ряд для характеристического функционала. Будем искать решение задачи (3), (4) в виде степенного ряда:

$$
\begin{aligned}
Y= & Y_{0}\left(v_{1}, v_{2}, w\right)+\sum_{k=1}^{\infty} \frac{i^{k}}{k !} \int \ldots \int Y_{k}\left(v_{1}, v_{2}, w, s_{1}, \ldots, s_{k}, x_{1}, \ldots, x_{k}\right) \\
& \times z\left(s_{1}, x_{1}\right) \ldots z\left(s_{k}, x_{k}\right) d s_{1} \ldots d s_{k} d x_{1} \ldots d x_{k}
\end{aligned}
$$

где интегралы по переменным $s_{1}, \ldots, s_{k}$ вычисляются по промежутку $T$, а по переменным $x_{1}, \ldots, x_{k}-$ по $\mathbb{R}$, отображения $Y_{k}$ симметричны по переменным $\left(s_{i}, x_{i}\right), \quad i=$ $1,2, \ldots, k$. Полагая в этом равенстве $z=0$, из определения $Y$ и $\varphi$ получаем

$$
Y\left(t, x, v_{1}, v_{2}, w, 0\right)=\varphi\left(v_{1}, v_{2}, w\right)=Y_{0}\left(v_{1}, v_{2}, w\right)
$$


Подставим разложение для $Y$ в уравнение (3), получим

$$
\begin{aligned}
\sum_{k=1}^{\infty} & \frac{i^{k}}{(k-1) !} \int \ldots \int \frac{\partial}{\partial t} Y_{k}\left(v_{1}, v_{2}, w, t, s_{2}, \ldots, s_{k}, x, x_{2}, \ldots, x_{k}\right) \\
& \times z\left(s_{2}, x_{2}\right) \ldots z\left(s_{k}, x_{k}\right) d s_{2} \ldots d s_{k} d x_{2} \ldots d x_{k} \\
= & -i \sum_{k=1}^{\infty} \frac{i^{k}}{(k-1) !} \int \ldots \int \frac{\delta}{\delta v_{1}(t)} \frac{\partial}{\partial x} Y_{k}\left(v_{1}, v_{2}, w, t, s_{2}, \ldots, s_{k}, x, x_{2}, \ldots, x_{k}\right) \\
& \times z\left(s_{2}, x_{2}\right) \ldots z\left(s_{k}, x_{k}\right) d s_{2} \ldots d s_{k} d x_{2} \ldots d x_{k} \\
& -i \sum_{k=1}^{\infty} \frac{i^{k}}{(k-1) !} \int \ldots \int \frac{\delta}{\delta v_{2}(t)} \frac{\partial^{2}}{\partial x^{2}} Y_{k}\left(v_{1}, v_{2}, w, t, s_{2}, \ldots, s_{k}, x, x_{2}, \ldots, x_{k}\right) \\
& \times z\left(s_{2}, x_{2}\right) \ldots z\left(s_{k}, x_{k}\right) d s_{2} \ldots d s_{k} d x_{2} \ldots d x_{k} \\
& +\frac{\delta Y_{0}\left(v_{1}, v_{2}, w\right)}{\delta w(t, x)}+\sum_{k=1}^{\infty} \frac{i^{k}}{k !} \int \ldots \int \frac{\delta}{\delta w(t, x)} Y_{k}\left(v_{1}, v_{2}, w, s_{1}, \ldots, s_{k}, x_{1}, \ldots, x_{k}\right) \\
& \times z\left(s_{1}, x_{1}\right) \ldots z\left(s_{k}, x_{k}\right) d s_{1} \ldots d s_{k} d x_{1} \ldots d x_{k} .
\end{aligned}
$$

Приравнивая степени по переменной $z$, получим

$$
\begin{aligned}
\frac{\partial}{\partial t} Y_{k} & \left(v_{1}, v_{2}, w, t, s_{2}, \ldots, s_{k}, x, x_{2}, \ldots, x_{k}\right) z\left(s_{2}, x_{2}\right) \ldots z\left(s_{k}, x_{k}\right) \\
= & -i \frac{\delta}{\delta v_{1}(t)} \frac{\partial}{\partial x} Y_{k}\left(v_{1}, v_{2}, w, t, s_{2}, \ldots, s_{k}, x, x_{2}, \ldots, x_{k}\right) z\left(s_{2}, x_{2}\right) \ldots z\left(s_{k}, x_{k}\right) \\
& -i \frac{\delta}{\delta v_{2}(t)} \frac{\partial^{2}}{\partial x^{2}} Y_{k}\left(v_{1}, v_{2}, w, t, s_{2}, \ldots, s_{k}, x, x_{2}, \ldots, x_{k}\right) z\left(s_{2}, x_{2}\right) \ldots z\left(s_{k}, x_{k}\right) \\
& -i \frac{\delta}{\delta w(t, x)} Y_{k-1}\left(v_{1}, v_{2}, w, s_{2}, \ldots, s_{k}, x_{2}, \ldots, x_{k}\right) z\left(s_{2}, x_{2}\right) \ldots z\left(s_{k}, x_{k}\right)
\end{aligned}
$$

при $k=1,2, \ldots$ и при всех $z\left(s_{2}, x_{2}\right), \ldots, z\left(s_{k}, x_{k}\right) \in L_{1}(T \times \mathbb{R})$. Это равносильно равенствам

$$
\begin{aligned}
\frac{\partial}{\partial t} Y_{k}\left(v_{1}, v_{2}, w, t, s_{2}, \ldots, s_{k}, x, x_{2}, \ldots, x_{k}\right) \\
=-i \frac{\delta}{\delta v_{1}(t)} \frac{\partial}{\partial x} Y_{k}\left(v_{1}, v_{2}, w, t, s_{2}, \ldots, s_{k}, x, x_{2}, \ldots, x_{k}\right) \\
\quad-i \frac{\delta}{\delta v_{2}(t)} \frac{\partial^{2}}{\partial x^{2}} Y_{k}\left(v_{1}, v_{2}, w, t, s_{2}, \ldots, s_{k}, x, x_{2}, \ldots, x_{k}\right) \\
\quad-i \frac{\delta}{\delta w(t, x)} Y_{k-1}\left(v_{1}, v_{2}, w, s_{2}, \ldots, s_{k}, x_{2}, \ldots, x_{k}\right)
\end{aligned}
$$

при $k=1,2, \ldots$ 
Вычислим вариационную производную $(k-1)$-го порядка по $z$ от выражения $(4)$ в точке $\left(v_{1}, v_{2}, w, 0, t_{0}, \ldots, t_{0}, x_{1}, x_{2}, \ldots, x_{k}\right)$. Учитывая независимость случайного процесса $u_{0}$ от $\varepsilon_{1}, \varepsilon_{2}, f$, получим

$$
\begin{aligned}
& i^{k} Y_{k}\left(v_{1}, v_{2}, 0, t_{0}, \ldots, t_{0}, x_{1}, x_{2}, \ldots, x_{k}\right) \\
& \quad=i^{k} \mathrm{M}\left(u_{0}\left(x_{1}\right) u_{0}\left(x_{2}\right) \ldots u_{0}\left(x_{k}\right) e_{1}\left(v_{1}, v_{2}, w, 0\right)\right) \\
& \quad=i^{k} \mathrm{M}\left(u_{0}\left(x_{1}\right) u_{0}\left(x_{2}\right) \ldots u_{0}\left(x_{k}\right)\right) \varphi\left(v_{1}, v_{2}, w\right), \quad k=1,2, \ldots,
\end{aligned}
$$

T. e.

$$
\begin{aligned}
& Y_{k}\left(v_{1}, v_{2}, 0, t_{0}, \ldots, t_{0}, x_{1}, x_{2}, \ldots, x_{k}\right) \\
& \quad=\mathrm{M}\left(u_{0}\left(x_{1}\right) u_{0}\left(x_{2}\right) \ldots u_{0}\left(x_{k}\right)\right) \varphi\left(v_{1}, v_{2}, w\right), \quad k=1,2, \ldots .
\end{aligned}
$$

Получили рекуррентную последовательность детерминированных задач (5), (6) для нахождения коэффициентов $Y_{k}$.

Если $Y\left(v_{1}, v_{2}, w, z\right)$ - характеристический функционал случайных процессов $\varepsilon_{1}, \varepsilon_{2}$, $f, u$, то

$$
\begin{aligned}
& Y_{k}\left(0,0,0, s_{1}, s_{2}, \ldots, s_{k}, x_{1}, x_{2}, \ldots, x_{k}\right) \\
& \quad=\left.i^{-k} \frac{\delta^{k} Y\left(v_{1}, v_{2}, w, z\right)}{\delta z\left(s_{1}, x_{1}\right) \delta z\left(s_{2}, x_{2}\right) \ldots \delta z\left(s_{k}, x_{k}\right)}\right|_{v_{1}=0, v_{2}=0, w=0, z=0} \\
& \quad=\operatorname{M}\left(u\left(s_{1}, x_{1}\right) u\left(s_{2}, x_{2}\right) \ldots u\left(s_{k}, x_{k}\right)\right) .
\end{aligned}
$$

Последнее является основанием для следуюшего определения.

ОПРЕДЕЛЕНИЕ. Пусть $Y_{k}\left(v_{1}, v_{2}, w, s_{1}, s_{2}, \ldots, s_{k}, x_{1}, x_{2}, \ldots, x_{k}\right)$ является симметрическим по переменным $s_{i}, x_{i}, i=1,2, \ldots, k$, решением, в смысле обобшенных функций (в классическом смысле), задачи (5), (6); тогда $Y_{k}\left(0,0,0, s_{1}, s_{2}, \ldots, s_{k}, x_{1}, x_{2}, \ldots, x_{k}\right)$ называется моментной функцией $k$-го порядка решения задачи (1), (2), в смысле обобщенных функций (в классическом смысле).

4. Решение дифференциального уравнения третьего порядка с обычными и вариационными производными. Уравнения (5) одного типа. Имеет смысл сначала исследовать этот тип уравнений. Рассмотрим задачу с начальным условием

$$
\begin{aligned}
& \frac{\partial}{\partial t} y\left(v_{1}, v_{2}, t, x\right)=-i \frac{\delta}{\delta v_{1}(t)} \frac{\partial}{\partial x} y\left(v_{1}, v_{2}, t, x\right) \\
&-i \frac{\delta}{\delta v_{2}(t)} \frac{\partial^{2}}{\partial x^{2}} y\left(v_{1}, v_{2}, t, x\right)+b\left(v_{1}, v_{2}, t, x\right) \\
& y\left(v_{1}, v_{2}, t_{0}, x\right)=y_{0}\left(v_{1}, v_{2}, x\right)
\end{aligned}
$$


где $v_{1} \in L_{1}(T), v_{2} \in L_{1}(T), t \in T, x \in \mathbb{R}, y: L_{1}(T) \times L_{1}(T) \times T \times \mathbb{R} \rightarrow \mathbb{C}$ - искомое отображение, $b$ и $y_{0}$ заданы.

В дальнейшем $\chi(\tau, t, \cdot)$ обозначает характеристическую функцию отрезка $[\tau, t]$, т. е. $\chi(\tau, t, s)=$ 1 при $s \in[\tau, t]$ и $\chi(\tau, t, s)=0$ в противном случае.

В формулировке следуюшей теоремы отображение $y_{0}$ и его производные вычисляются в точках $\left(v_{1}-\xi \chi\left(t_{0}, t, \cdot\right), v_{2}+i \xi^{2} \chi\left(t_{0}, t, \cdot\right), x\right)$, а отображение $b$ и его производные вычисляются в точках $\left(v_{1}-\xi \chi(\tau, t, \cdot), v_{2}+i \xi^{2} \chi(\tau, t, \cdot), \tau, x\right)$.

Теорема 1. Пусть существует окрестность $U(r)$ нуля радиуса $r$ в $L_{1}(T) \times$ $L_{1}(T)$ такая, что при всех $\left(v_{1}, v_{2}\right) \in U(r)$ функции

$$
\begin{gathered}
|y|,\left|\frac{\delta y_{0}}{\delta v_{1}(t)}\right|,\left|\frac{\delta y_{0}}{\delta v_{2}(t)}\right|,\left|F_{x}\left[\frac{\delta y_{0}}{\delta v_{1}(t)}\right](\xi)\right|,\left|F_{x}\left[\frac{\delta y_{0}}{\delta v_{2}(t)}\right](\xi)\right|, \\
\left|\xi^{2} F_{x}\left[y_{0}\right](\xi)\right|,\left|\xi F_{x}\left[\frac{\delta y_{0}}{\delta v_{1}(t)}\right](\xi)\right|,\left|\xi F_{x}\left[\frac{\delta y_{0}}{\delta v_{2}(t)}\right](\xi)\right|,\left|\xi^{2} F_{x}\left[\frac{\delta y_{0}}{\delta v_{2}(t)}\right](\xi)\right|, \\
|b|,\left|\frac{\delta b}{\delta v_{1}(t)}\right|,\left|\frac{\delta b}{\delta v_{2}(t)}\right|,\left|F_{x}\left[\frac{\delta b}{\delta v_{1}(t)}\right](\xi)\right|,\left|F_{x}\left[\frac{\delta b}{\delta v_{2}(t)}\right](\xi)\right| \\
\left|\xi^{2} F_{x}[b](\xi)\right|,\left|\xi F_{x}\left[\frac{\delta b}{\delta v_{1}(t)}\right](\xi)\right|,\left|\xi F_{x}\left[\frac{\delta b}{\delta v_{2}(t)}\right](\xi)\right|,\left|\xi^{2} F_{x}\left[\frac{\delta b}{\delta v_{2}(t)}\right](\xi)\right|
\end{gathered}
$$

при $t \in T, \tau \in T$ ограничены суммируемыми на $\mathbb{R}$ функциями. Тогда

$$
\begin{gathered}
y\left(v_{1}, v_{2}, t, x\right)=F_{\xi}^{-1}\left[F_{x}\left[y_{0}\left(v_{1}-\xi \chi\left(t_{0}, t, \cdot\right), v_{2}+i \xi^{2} \chi\left(t_{0}, t, \cdot\right), x\right)\right](\xi)\right](x) \\
+\int_{t_{0}}^{t} F_{\xi}^{-1}\left[F_{x}\left[b\left(v_{1}-\xi \chi(\tau, t, \cdot), v_{2}+i \xi^{2} \chi(\tau, t, \cdot), \tau, x\right)\right](\xi)\right](x) d \tau
\end{gathered}
$$

является единственным решением задачи (7), (8).

ДокАЗАТЕльСтво. Предположим, что сушествует преобразование Фурье по переменной $x$ решения задачи $(7),(8)$. Применяя преобразование Фурье к уравнениям (7), (8), получаем

$$
\begin{gathered}
\frac{\partial}{\partial t} F_{x}\left[y\left(v_{1}, v_{2}, t, x\right)\right](\xi)=-i \frac{\delta}{\delta v_{1}(t)}(-i \xi) F_{x}\left[y\left(v_{1}, v_{2}, t, x\right)\right](\xi) \\
-i \frac{\delta}{\delta v_{2}(t)}(-i \xi)^{2} F_{x}\left[y\left(v_{1}, v_{2}, t, x\right)\right](\xi)+F_{x}\left[b\left(v_{1}, v_{2}, t, x\right)\right](\xi), \\
F_{x}\left[y\left(v_{1}, v_{2}, t_{0}, x\right)\right](\xi)=F_{x}\left[y_{0}\left(v_{1}, v_{2}, x\right)\right](\xi) .
\end{gathered}
$$

Эта задача имеет вид

$$
\begin{gathered}
\frac{\partial Y\left(v_{1}, v_{2}, t\right)}{\partial t}=a_{1}(t) \frac{\delta Y\left(v_{1}, v_{2}, t\right)}{\delta v_{1}(t)}+a_{2}(t) \frac{\delta Y\left(v_{1}, v_{2}, t\right)}{\delta v_{2}(t)}+B\left(v_{1}, v_{2}, t\right) \\
Y\left(v_{1}, v_{2}, t_{0}\right)=Y_{0}\left(v_{1}, v_{2}\right) .
\end{gathered}
$$


Такая задача изучена в [13]. Оказывается, если функции $a_{i}: T \rightarrow \mathbb{C}, i=1,2$, непрерывны и сушествуют вариационные производные

$$
\frac{\delta Y_{0}\left(v_{1}+a_{1} \chi\left(t_{0}, t, \cdot\right), v_{2}+a_{2} \chi\left(t_{0}, t, \cdot\right)\right)}{\delta v_{i}(t)}, \frac{\delta B\left(v_{1}+a_{1} \chi(\tau, t, \cdot), v_{2}+a_{2} \chi(\tau, t, \cdot), \tau\right)}{\delta v_{i}(t)},
$$

$i=1,2, t_{0} \leqslant \tau \leqslant t$, то

$$
\begin{aligned}
Y\left(v_{1}, v_{2}, t\right)= & Y_{0}\left(v_{1}+a_{1} \chi\left(t_{0}, t, \cdot\right), v_{2}+a_{2} \chi\left(t_{0}, t, \cdot\right)\right) \\
& +\int_{t_{0}}^{t} B\left(v_{1}+a_{1} \chi(\tau, t, \cdot), v_{2}+a_{2} \chi(\tau, t, \cdot), \tau\right) d \tau
\end{aligned}
$$

является единственным решением последней задачи. (Это можно проверить, вычислив производную $\partial Y\left(v_{1}, v_{2}, t\right) / \partial t$ и подставив в уравнение.)

Воспользовавшись этим результатом, находим решение задачи (10), (11)

$$
\begin{aligned}
F_{x}\left[y\left(v_{1}, v_{2}, t, x\right)\right](\xi)= & F_{x}\left[y_{0}\left(v_{1}-\xi \chi\left(t_{0}, t, \cdot\right), v_{2}+i \xi^{2} \chi\left(t_{0}, t, \cdot\right), x\right)\right](\xi) \\
& +\int_{t_{0}}^{t} F_{x}\left[b\left(v_{1}-\xi \chi(\tau, t, \cdot), v_{2}+i \xi^{2} \chi(\tau, t, \cdot), \tau, x\right)\right](\xi) d \tau .
\end{aligned}
$$

Для получения формулы (9) нужно применить к последнему равенству обратное преобразование Фурье.

Подставим (9) в уравнение (10). Предположения теоремы о сушествовании суммируемых мажорант обеспечивают сушествование вариационных производныхпо $v_{i}$, $i=1,2$, и смешанных производных, а также обеспечивают дифференшируемость под знаками интегралов. При этом получается тождество, что и доказывает теорему.

5. Математическое ожидание решения. Для нахождения математического ожидания решения задачи (1), (2) выпишем задачу (5), (6) при $k=1$ :

$$
\begin{gathered}
\frac{\partial}{\partial t} Y_{1}\left(v_{1}, v_{2}, w, t, x\right)=-i \frac{\delta}{\delta v_{1}(t)} \frac{\partial}{\partial x} Y_{1}\left(v_{1}, v_{2}, w, t, x\right) \\
-i \frac{\delta}{\delta v_{2}(t)} \frac{\partial^{2}}{\partial x^{2}} Y_{1}\left(v_{1}, v_{2}, w, t, x\right)-i \frac{\delta}{\delta w(t, x)} \varphi\left(v_{1}, v_{2}, w\right), \\
Y_{1}\left(v_{1}, v_{2}, w, t_{0}, x\right)=\mathrm{M} u_{0}(x) \varphi\left(v_{1}, v_{2}, w\right) .
\end{gathered}
$$

Теорема 2. Пусть функция $\mathrm{M} u_{0}(x)$ суммируема на $\mathbb{R}$ и при мальх $w \in L_{1}(T \times \mathbb{R})$ выполняются условия предыдущей теоремь. Тогда

$$
\begin{aligned}
& Y_{1}\left(v_{1}, v_{2}, w, t, x\right) \\
& \quad=\mathrm{M} u_{0}(x) \stackrel{x}{*} F_{\xi}^{-1}\left[\varphi\left(v_{1}-\xi \chi\left(t_{0}, t, \cdot\right), v_{2}+i \xi^{2} \chi\left(t_{0}, t, \cdot\right), w\right)\right](x) \\
& \quad-i \int_{t_{0}}^{t} F_{\xi}^{-1}\left[F_{x}\left[\frac{\delta \varphi\left(v_{1}-\xi \chi(\tau, t, \cdot), v_{2}+i \xi^{2} \chi(\tau, t, \cdot), w\right)}{\delta w(\tau, x)}\right](\xi)\right](x) d \tau
\end{aligned}
$$

является единственным решением задачи (12), (13). 
ДокАЗАТЕЛьство. По формуле (9) получаем решение задачи (12), (13)

$$
\begin{aligned}
Y_{1}= & F_{\xi}^{-1}\left[F_{x}\left[\mathrm{M} u_{0}(x) \varphi\left(v_{1}-\xi \chi\left(t_{0}, t, \cdot\right), v_{2}+i \xi^{2} \chi\left(t_{0}, t, \cdot\right), w\right)\right](x)\right. \\
& -i \int_{t_{0}}^{t} F_{\xi}^{-1}\left[F_{x}\left[\frac{\delta \varphi\left(v_{1}-\xi \chi(\tau, t, \cdot), v_{2}+i \xi^{2} \chi(\tau, t, \cdot), w\right)}{\delta w(\tau, x)}\right](\xi)\right](x) d \tau .
\end{aligned}
$$

Далее,

$$
\begin{aligned}
& F_{x}\left[\operatorname{M} u_{0}(x) \varphi\left(v_{1}-\xi \chi\left(t_{0}, t, \cdot\right), v_{2}+i \xi^{2} \chi\left(t_{0}, t, \cdot\right), w\right)\right](\xi) \\
& \quad=F_{x}\left[\operatorname{Mu} u_{0}(x)\right](\xi) \varphi\left(v_{1}-\xi \chi\left(t_{0}, t, \cdot\right), v_{2}+i \xi^{2} \chi\left(t_{0}, t, \cdot\right), w\right) .
\end{aligned}
$$

Поскольку обратное преобразование Фурье от произведения равно свертке обратных преобразований Фурье от сомножителей, то получаем (14). Теорема доказана.

Теорема 3. При выполнении условий теоремь 2

$$
\begin{aligned}
\mathrm{M} u(t, x)= & \mathrm{M} u_{0}(x) \stackrel{x}{*} F_{\xi}^{-1}\left[\varphi\left(-\xi \chi\left(t_{0}, t, \cdot\right), i \xi^{2} \chi\left(t_{0}, t, \cdot\right), 0\right)\right](x) \\
& -i \int_{t_{0}}^{t} F_{\xi}^{-1}\left[F_{x}\left[\frac{\delta \varphi\left(-\xi \chi(\tau, t, \cdot), i \xi^{2} \chi(\tau, t, \cdot), 0\right)}{\delta w(\tau, x)}\right](\xi)\right](x) d \tau
\end{aligned}
$$

является математическим ожиданием решения задачи (1), (2).

ДоКАЗАТЕЛЬСТво получается подстановкой $v_{1}=0, v_{2}=0, w=0$ в формулу (14).

6. Случай независимых процессов $f$ и $\varepsilon_{1}, \varepsilon_{2}$. Формула (15) является достаточно общей, не требуется даже независимости случайных процессов $f, \varepsilon_{1}, \varepsilon_{2}$. При независимых процессах можно продвинуться дальше. Пусть $\psi\left(v_{1}, v_{2}\right)$ - характеристический функционал процессов $\varepsilon_{1}, \varepsilon_{2}$.

Теорема 4. Если случайный прочесс $f$ не зависит от случайных прочессов $\varepsilon_{1}$, $\varepsilon_{2}$ и выполнены условия теоремы 2 , то

$$
\begin{aligned}
\mathrm{M} u(t, x)= & \mathrm{M} u_{0}(x) \stackrel{x}{*} F_{\xi}^{-1}\left[\psi\left(-\xi \chi\left(t_{0}, t, \cdot\right), i \xi^{2} \chi\left(t_{0}, t, \cdot\right)\right)\right](x) \\
& +\int_{t_{0}}^{t} F_{\xi}^{-1}\left[\psi\left(-\xi \chi(\tau, t, \cdot), i \xi^{2} \chi(\tau, t, \cdot)\right)\right](x) \stackrel{x}{*} \mathrm{M} f(\tau, x) d \tau
\end{aligned}
$$

яв.лется математическим ожиданием решения задачи (1), (2). 
ДокАЗАтЕльство. При независимых случайных процессах $f$ и $\varepsilon_{1}, \varepsilon_{2}$ характеристический функционал $\varphi$ имеет вид $\varphi\left(v_{1}, v_{2}, w\right)=\psi\left(v_{1}, v_{2}\right) \varphi_{f}(w)$, где $\varphi_{f}(w)$ - характеристический функционал случайного процесса $f$. При этом

$$
\begin{aligned}
\frac{\delta \varphi\left(-\xi \chi(\tau, t, \cdot), i \xi^{2} \chi(\tau, t, \cdot), 0\right)}{\delta w(\tau, x)} & =\left.\psi\left(-\xi \chi(\tau, t, \cdot), i \xi^{2} \chi(\tau, t, \cdot)\right) \frac{\delta \varphi_{f}(w)}{\delta w(\tau, x)}\right|_{w=0} \\
& =i \psi\left(-\xi \chi(\tau, t, \cdot), i \xi^{2} \chi(\tau, t, \cdot)\right) \operatorname{M} f(\tau, x) .
\end{aligned}
$$

Далее,

$$
\begin{aligned}
F_{\xi}^{-1}\left[F_{x}\right. & {\left.\left[\psi\left(-\xi \chi(\tau, t, \cdot), i \xi^{2} \chi(\tau, t, \cdot)\right) \operatorname{M} f(\tau, x)\right](\xi)\right](x) } \\
& =F_{\xi}^{-1}\left[\psi\left(-\xi \chi(\tau, t, \cdot), i \xi^{2} \chi(\tau, t, \cdot)\right) F_{x}[\operatorname{M} f(\tau, x)](\xi)\right](x) \\
& =F_{\xi}^{-1}\left[\psi\left(-\xi \chi(\tau, t, \cdot), i \xi^{2} \chi(\tau, t, \cdot)\right)\right](x) \stackrel{x}{*} \operatorname{M} f(\tau, x) .
\end{aligned}
$$

Подставляя эти соотношения в (15), получаем (16). Теорема доказана.

7. Случай гауссовских процессов $\varepsilon_{1}, \varepsilon_{2}$. Рассмотрим наиболее частовстречающийся случай гауссовских процессов $\varepsilon_{1}, \varepsilon_{2}$, которые заданы характеристическим функционалом

$$
\begin{aligned}
\psi\left(v_{1}, v_{2}\right)= & \exp \left(i \int_{T} \mathrm{M} \varepsilon_{1}(s) v_{1}(s) d s-\frac{1}{2} \int_{T} \int_{T} b_{1}\left(s_{1}, s_{2}\right) v_{1}\left(s_{1}\right) v_{1}\left(s_{2}\right) d s_{1} d s_{2}\right. \\
& \left.+i \int_{T} \mathrm{M} \varepsilon_{2}(s) v_{2}(s) d s-\frac{1}{2} \int_{T} \int_{T} b_{2}\left(s_{1}, s_{2}\right) v_{2}\left(s_{1}\right) v_{2}\left(s_{2}\right) d s_{1} d s_{2}\right),
\end{aligned}
$$

где $b_{1}\left(s_{1}, s_{2}\right), b_{2}\left(s_{1}, s_{2}\right)$ - ковариационные функции процессов $\varepsilon_{1}$ и $\varepsilon_{2}$ соответственно.

Теорема 5. Пусть случайный процесс $f(t, x)$ не зависит от случайных процессов $\varepsilon_{1}, \varepsilon_{2}$, математическое ожсидание $\mathrm{M} f(t, x)$ локально суммируемо, $b_{i} \in$ $L_{\infty}(T \times T), \quad i=1,2$. Пусть при всех $t \in T$ и всех $s_{1} \in T, \quad t_{0} \leqslant \tau<t \leqslant t_{1}$, за исключением множества нулевой меры, выполняется условие

$$
\int_{\tau}^{t} b_{1}\left(s_{1}, s_{2}\right) d s_{2}+2 \mathrm{M} \varepsilon_{2}\left(s_{1}\right)>0 .
$$


Тогда

$$
\begin{aligned}
\mathrm{M} u(t, x)=( & \left.2 \pi \int_{t_{0}}^{t} \int_{t_{0}}^{t} b_{1}\left(s_{1}, s_{2}\right) d s_{1} d s_{2}+4 \pi \int_{t_{0}}^{t} \mathrm{M} \varepsilon_{2}(s) d s\right)^{-0.5} \\
& \times \exp \left(-\frac{\left(x+\int_{t_{0}}^{t} \mathrm{M} \varepsilon_{1}(s) d s\right)^{2}}{2 \int_{t_{0}}^{t} \int_{t_{0}}^{t} b_{1}\left(s_{1}, s_{2}\right) d s_{1} d s_{2}+4 \int_{t_{0}}^{t} \mathrm{M} \varepsilon_{2}(s) d s}\right) \\
& * \sum_{k=0}^{\infty} \frac{\left(\int_{t_{0}}^{t} \int_{t_{0}}^{t} b_{2}\left(s_{1}, s_{2}\right) d s_{1} d s_{2}\right)^{k}}{2^{k} k !} \frac{d^{4 k}}{d x^{4 k}} \mathrm{M} u_{0}(x) \\
& +\int_{t_{0}}^{t}\left(2 \pi \int_{\tau}^{t} \int_{\tau}^{t} b_{1}\left(s_{1}, s_{2}\right) d s_{1} d s_{2}+4 \pi \int_{\tau}^{t} \mathrm{M} \varepsilon_{2}(s) d s\right)^{-0.5} \\
& \times \exp \left(-\frac{\left(x+\int_{\tau}^{t} \mathrm{M} \varepsilon_{1}(s) d s\right)^{2}}{2 \int_{\tau}^{t} \int_{\tau}^{t} b_{1}\left(s_{1}, s_{2}\right) d s_{1} d s_{2}+4 \int_{\tau}^{t} \mathrm{M} \varepsilon_{2}(s) d s}\right) \\
& * \sum_{k=0}^{\infty} \frac{\left(\int_{\tau}^{t} \int_{\tau}^{t} b_{2}\left(s_{1}, s_{2}\right) d s_{1} d s_{2}\right)^{k}}{2^{k} k !} \frac{\partial^{4 k}}{\partial x^{4 k}} \mathrm{Mf} f(\tau, x) d \tau
\end{aligned}
$$

является математическим ожиданием (в смысле обобщенных функиий) решения задачи (1), (2).

ДокАЗАТЕЛЬСтво. Для нахождения $\mathrm{M} u(t, x)$ воспользуемся формулой $(16)$, при этом

$$
\begin{aligned}
F_{\xi}^{-1}[ & \left.\psi\left(-\xi \chi\left(t_{0}, t, \cdot\right), i \xi^{2} \chi\left(t_{0}, t, \cdot\right)\right)\right]=F_{\xi}^{-1}\left[\operatorname { e x p } \left(-i \xi \int_{t_{0}}^{t} \mathrm{M} \varepsilon_{1}(s) d s\right.\right. \\
& -\frac{\xi^{2}}{2}\left(\int_{t_{0}}^{t} \int_{t_{0}}^{t} b_{1}\left(s_{1}, s_{2}\right) d s_{1} d s_{2}+2 \int_{t_{0}}^{t} \mathrm{M} \varepsilon_{2}(s) d s\right) \\
& \left.\left.+\frac{\xi^{4}}{2} \int_{t_{0}}^{t} \int_{t_{0}}^{t} b_{2}\left(s_{1}, s_{2}\right) d s_{1} d s_{2}\right)\right] \\
= & F_{\xi}^{-1}\left[\exp \left(-i \xi \int_{t_{0}}^{t} \mathrm{M} \varepsilon_{1}(s) d s\right)\right] \\
& * F_{\xi}^{-1}\left[\exp \left(-\frac{\xi^{2}}{2}\left(\int_{t_{0}}^{t}\left(\int_{t_{0}}^{t} b_{1}\left(s_{1}, s_{2}\right) d s_{2}+2 \mathrm{M} \varepsilon_{2}\left(s_{1}\right)\right) d s_{1}\right)\right)\right] \\
& * F_{\xi}^{-1}\left[\exp \left(\frac{\xi^{4}}{2} \int_{t_{0}}^{t} \int_{t_{0}}^{t} b_{2}\left(s_{1}, s_{2}\right) d s_{1} d s_{2}\right)\right]
\end{aligned}
$$

Далее находим [16, с. 145]

$$
F_{\xi}^{-1}\left[\exp \left(-i \xi \int_{t_{0}}^{t} \mathrm{M} \varepsilon_{1}(s) d s\right)\right]=\delta\left(-x-\int_{t_{0}}^{t} \mathrm{M} \varepsilon_{1}(s) d s\right)
$$


Так как выполнено условие (17), то

$$
\begin{aligned}
F_{\xi}^{-1}\left[\exp \left(-\frac{\xi^{2}}{2}\left(\int_{t_{0}}^{t}\left(\int_{t_{0}}^{t} b_{1}\left(s_{1}, s_{2}\right) d s_{2}+2 \mathrm{M} \varepsilon_{2}\left(s_{1}\right)\right) d s_{1}\right)\right)\right] \\
=\left(2 \pi \int_{t_{0}}^{t} \int_{t_{0}}^{t} b_{1}\left(s_{1}, s_{2}\right) d s_{1} d s_{2}+4 \pi \int_{t_{0}}^{t} \mathrm{M} \varepsilon_{2}\left(s_{1}\right) d s_{1}\right)^{-0.5} \\
\quad \times \exp \left(-\frac{x^{2}}{2 \int_{t_{0}}^{t} \int_{t_{0}}^{t} b_{1}\left(s_{1}, s_{2}\right) d s_{1} d s_{2}+4 \int_{t_{0}}^{t} \mathrm{M} \varepsilon_{2}\left(s_{1}\right) d s_{1}}\right), \\
F_{\xi}^{-1}\left[\exp \left(\frac{\xi^{4}}{2} \int_{t_{0}}^{t} \int_{t_{0}}^{t} b_{2}\left(s_{1}, s_{2}\right) d s_{1} d s_{2}\right)\right] \\
=\sum_{k=0}^{\infty} \frac{1}{2^{k} k !}\left(\int_{t_{0}}^{t} \int_{t_{0}}^{t} b_{2}\left(s_{1}, s_{2}\right) d s_{1} d s_{2}\right)^{k}\left(-i \frac{d}{d x}\right)^{4 k} \delta(-x) \\
=\sum_{k=0}^{\infty} \frac{1}{2^{k} k !}\left(\int_{t_{0}}^{t} \int_{t_{0}}^{t} b_{2}\left(s_{1}, s_{2}\right) d s_{1} d s_{2}\right)^{k} \frac{d^{4 k}}{d x^{4 k}} \delta(x)
\end{aligned}
$$

где $\delta$ - дельта-функция.

Далее,

$$
\begin{aligned}
\delta(-x & \left.-\int_{t_{0}}^{t} \mathrm{M} \varepsilon_{1}(s) d s\right) \\
& *\left(2 \pi \int_{t_{0}}^{t} \int_{t_{0}}^{t} b_{1}\left(s_{1}, s_{2}\right) d s_{1} d s_{2}+4 \pi \int_{t_{0}}^{t} \mathrm{M} \varepsilon_{2}\left(s_{1}\right) d s_{1}\right)^{-0.5} \\
& \times \exp \left(-\frac{x^{2}}{2 \int_{t_{0}}^{t} \int_{t_{0}}^{t} b_{1}\left(s_{1}, s_{2}\right) d s_{1} d s_{2}+4 \int_{t_{0}}^{t} \mathrm{M} \varepsilon_{2}\left(s_{1}\right) d s_{1}}\right) \\
= & \int_{-\infty}^{\infty} \delta\left(\eta+\int_{t_{0}}^{t} \mathrm{M} \varepsilon_{1}(s) d s\right) \\
& \times\left(2 \pi \int_{t_{0}}^{t} \int_{t_{0}}^{t} b_{1}\left(s_{1}, s_{2}\right) d s_{1} d s_{2}+4 \pi \int_{t_{0}}^{t} \mathrm{M} \varepsilon_{2}\left(s_{1}\right) d s_{1}\right) \\
& \times \exp \left(-\frac{(x-\eta)^{2}}{2 \int_{t_{0}}^{t} \int_{t_{0}}^{t} b_{1}\left(s_{1}, s_{2}\right) d s_{1} d s_{2}+4 \int_{t_{0}}^{t} \mathrm{M} \varepsilon_{2}\left(s_{1}\right) d s_{1}}\right) \\
= & \left(2 \pi \int_{t_{0}}^{t} \int_{t_{0}}^{t} b_{1}\left(s_{1}, s_{2}\right) d s_{1} d s_{2}+4 \pi \int_{t_{0}}^{t} \mathrm{M} \varepsilon_{2}\left(s_{1}\right) d s_{1}\right) \\
& \times \exp \left(-\frac{\left(x+\int_{t_{0}}^{t} \mathrm{M} \varepsilon_{1}(s) d s\right)^{2}}{2 \int_{t_{0}}^{t} \int_{t_{0}}^{t} b_{1}\left(s_{1}, s_{2}\right) d s_{1} d s_{2}+4 \int_{t_{0}}^{t} \mathrm{M} \varepsilon_{2}\left(s_{1}\right) d s_{1}}\right) .
\end{aligned}
$$


Аналогично находим

$$
\begin{array}{r}
\mathrm{M} u_{0}(x) \stackrel{x}{*} \sum_{k=0}^{\infty} \frac{1}{2^{k} k !}\left(\int_{t_{0}}^{t} \int_{t_{0}}^{t} b_{2}\left(s_{1}, s_{2}\right) d s_{1} d s_{2}\right)^{k} \frac{d^{4 k}}{d x^{4 k}} \delta(x) \\
=\sum_{k=0}^{\infty} \frac{1}{2^{k} k !}\left(\int_{t_{0}}^{t} \int_{t_{0}}^{t} b_{2}\left(s_{1}, s_{2}\right) d s_{1} d s_{2}\right)^{k} \frac{d^{4 k}}{d x^{4 k}} \mathrm{M} u_{0}(x)
\end{array}
$$

Подставив эти значения в (16), получаем (18). Теорема доказана.

Приведем условия, при которых (18) является математическим ожиданием решения в классическом смысле.

Теорема 6. Пусть гауссовские случайные процессы $\varepsilon_{1}$, $\varepsilon_{2}$ не зависят от случайного процесса $f$, выполнено условие $(17), b_{1}, b_{2}$ суммируемы на $T \times T, \mathrm{M} u_{0}$ имеет производные любого порядка, $\mathrm{M} f(t, x)$ локально суммируемо и имеет частные производнье любого порядка по переменной $x$, существуют неотрицательнье постоянные $c, c_{1}, q$, при которых выполняются неравенства

$$
\begin{gathered}
\left|\frac{d^{4 k}}{d x^{4 k}} \mathrm{M} u_{0}(x)\right| \leqslant c k ! q^{k}, \quad\left|\frac{\partial^{4 k}}{\partial x^{4 k}} \mathrm{M} f(t, x)\right| \leqslant c_{1} k ! q^{k} \\
q \int_{T} \int_{T} b_{2}\left(s_{1}, s_{2}\right) d s_{1} d s_{2}<2 .
\end{gathered}
$$

Тогда (18) определяет математическое ожидание $\mathrm{M} u(t, x)$ решения задачи (1), $(2)$ и справедлива оценка

$$
\begin{aligned}
|\mathrm{M} u(t, x)| \leqslant & \frac{2 c}{2-q \int_{t_{0}}^{t} \int_{t_{0}}^{t} b_{2}\left(s_{1}, s_{2}\right) d s_{1} d s_{2}} \\
& \quad+2 c_{1} \int_{t_{0}}^{t}\left(2-q \int_{\tau}^{t} \int_{\tau}^{t} b_{2}\left(s_{1}, s_{2}\right) d s_{1} d s_{2}\right)^{-1} d \tau
\end{aligned}
$$


ДокАЗАТЕльство. Оцениваем выражение (18):

$$
\begin{aligned}
|\mathrm{M} u(t, x)| \leqslant & \int_{-\infty}^{\infty}\left(2 \pi \int_{t_{0}}^{t} \int_{t_{0}}^{t} b_{1}\left(s_{1}, s_{2}\right) d s_{1} d s_{2}+4 \pi \int_{t_{0}}^{t} \mathrm{M} \varepsilon_{2}(s) d s\right)^{-0.5} \\
& \times \exp \left(-\frac{\left(x-\eta+\int_{t_{0}}^{t} \mathrm{M} \varepsilon_{1}(s) d s\right)^{2}}{2 \int_{t_{0}}^{t} \int_{t_{0}}^{t} b_{1}\left(s_{1}, s_{2}\right) d s_{1} d s_{2}+4 \int_{t_{0}}^{t} \mathrm{M} \varepsilon_{2}(s) d s}\right) \\
& \times c \sum_{k=0}^{\infty} \frac{1}{2^{k}}\left(q \int_{t_{0}}^{t} \int_{t_{0}}^{t} b_{2}\left(s_{1}, s_{2}\right) d s_{1} d s_{2}\right)^{k} d \eta \\
& +\int_{t_{0}}^{t}\left(2 \pi \int_{\tau}^{t} \int_{\tau}^{t} b_{1}\left(s_{1}, s_{2}\right) d s_{1} d s_{2}+4 \pi \int_{\tau}^{t} \mathrm{M} \varepsilon_{2}(s) d s\right)^{-0.5} \\
& \times \int_{-\infty}^{\infty} \exp \left(-\frac{\left(x-\eta+\int_{\tau}^{t} \mathrm{M} \varepsilon_{1}(s) d s\right)^{2}}{2 \int_{\tau}^{t} \int_{\tau}^{t} b_{1}\left(s_{1}, s_{2}\right) d s_{1} d s_{2}+4 \int_{\tau}^{t} \mathrm{M} \varepsilon_{2}(s) d s}\right) \\
& \times c_{1} \sum_{k=0}^{\infty}\left(\frac{q}{2} \int_{\tau}^{t} \int_{\tau}^{t} b_{2}\left(s_{1}, s_{2}\right) d s_{1} d s_{2}\right)^{k} d \eta d \tau .
\end{aligned}
$$

Используя хорошо известное равенство

$$
\int_{-\infty}^{\infty} \exp \left(-x^{2} a^{2}\right) d x=\frac{\sqrt{\pi}}{a}, \quad a>0
$$

получаем

$$
\begin{aligned}
|\mathrm{M} u(t, x)| \leqslant & c \sum_{k=0}^{\infty}\left(\frac{q}{2} \int_{t_{0}}^{t} \int_{t_{0}}^{t} b_{2}\left(s_{1}, s_{2}\right) d s_{1} d s_{2}\right)^{k} \\
& +\int_{t_{0}}^{t} c_{1} \sum_{k=0}^{\infty}\left(\frac{q}{2} \int_{\tau}^{t} \int_{\tau}^{t} b_{2}\left(s_{1}, s_{2}\right) d s_{1} d s_{2}\right)^{k} \\
= & \frac{2 c}{2-q \int_{t_{0}}^{t} \int_{t_{0}}^{t} b_{2}\left(s_{1}, s_{2}\right) d s_{1} d s_{2}} \\
& +2 c_{1} \int_{t_{0}}^{t}\left(2-q \int_{\tau}^{t} \int_{\tau}^{t} b_{2}\left(s_{1}, s_{2}\right) d s_{1} d s_{2}\right)^{-1} d \tau
\end{aligned}
$$

Теорема доказана.

Заметим, что при $b_{1}=b_{2}=0$ коэффициенты $\varepsilon_{1}, \varepsilon_{2}$ являются детерминированными и равными $\mathrm{M} \varepsilon_{1}, \mathrm{M} \varepsilon_{2}$. Устремив в (18) $b_{1}$ и $b_{2}$ к нулю, в пределе получим формулу для 
математического ожидания решения задачи $(1),(2)$ с детерминированными $\varepsilon_{1}, \varepsilon_{2}$ :

$$
\begin{aligned}
\mathrm{M} u(t, x)= & \int_{-\infty}^{\infty}\left(4 \pi \int_{t_{0}}^{t} \mathrm{M} \varepsilon_{2}(s) d s\right)^{-0.5} \\
& \times \exp \left(-\frac{\left(x-\eta+\int_{t_{0}}^{t} \mathrm{M} \varepsilon_{1}(s) d s\right)^{2}}{4 \int_{t_{0}}^{t} \mathrm{M} \varepsilon_{2}(s) d s}\right) \mathrm{M} u_{0}(\eta) d \eta \\
& +\int_{t_{0}}^{t} \int_{-\infty}^{\infty}\left(4 \pi \int_{\tau}^{t} \mathrm{M} \varepsilon_{2}(s) d s\right)^{-0.5} \\
& \times \exp \left(-\frac{\left(x-\eta+\int_{\tau}^{t} \mathrm{M} \varepsilon_{1}(s) d s\right)^{2}}{4 \int_{\tau}^{t} \mathrm{M} \varepsilon_{2}(s) d s}\right) \mathrm{Mf}(\tau, \eta) d \eta d \tau
\end{aligned}
$$

При $\mathrm{M} \varepsilon_{1}=0$ отсюда получаем обобшение формулы Пуассона решения задачи Коши для уравнения теплопроводности на случай задачи со случайным начальным условием $u_{0}(x)$ и случайным возмушением $f(t, x)$.

8. Случай равномерно распределенного процесса и гауссовского процесса. Формула (16) позволяет рассматривать задачи со случайными процессами $\varepsilon_{1}, \varepsilon_{2}$, имеющими различные законы распределения.

Введем обозначения

$$
A(\tau, t)=\int_{\tau}^{t} a(s) d s, \quad B(\tau, t)=\int_{\tau}^{t} \mathrm{M} \varepsilon_{2}(s) d s .
$$

Теорема 7. Пусть случайный процесс $\varepsilon_{1}$ имеет равномерное распределение $c$ характеристическим функционалом

$$
\psi_{\varepsilon_{1}}(v)=\frac{\sin \int_{T} a(s) v(s) d s}{\int_{T} a(s) v(s) d s} \exp \left(i \int_{T} \mathrm{M} \varepsilon_{1}(s) v(s) d s\right), \quad a(t) \geqslant 0,
$$

случайный процесс $\varepsilon_{2}(t)$ имеет характеристический функционал

$$
\psi_{\varepsilon_{2}}(v)=\exp \left(i \int_{T} \mathrm{M} \varepsilon_{2}(s) v(s) d s-\frac{1}{2} \int_{T} \int_{T} b_{2}\left(s_{1}, s_{2}\right) v\left(s_{1}\right) v\left(s_{2}\right) d s_{1} d s_{2}\right),
$$

процессы $\varepsilon_{1}, \varepsilon_{2}, f$ независимь $и \mathrm{M} \varepsilon_{2}(t)>0$. Тогда

$$
\begin{aligned}
\mathrm{M} u(t, x)=\frac{1}{4} & \frac{1}{A\left(t_{0}, t\right)\left(\pi B\left(t_{0}, t\right)\right)^{0.5}} \int_{-B\left(t_{0}, t\right)-A\left(t_{0}, t\right)}^{-B\left(t_{0}, t\right)+A\left(t_{0}, t\right)} \exp \left(-\frac{(x-\eta)^{2}}{4 B\left(t_{0}, t\right)}\right) d \eta \\
& \quad * \sum_{k=0}^{\infty} \frac{1}{2^{k} k !}\left(\int_{t_{0}}^{t} \int_{t_{0}}^{t} b_{2}\left(s_{1}, s_{2}\right) d s_{1} d s_{2}\right)^{k} \frac{d^{4 k}}{d x^{4 k}} \mathrm{M} u_{0}(x) \\
& +\int_{t_{0}}^{t} \frac{1}{4 A(\tau, t)(\pi B(\tau, t))^{0.5}} \int_{-B(\tau, t)-A(\tau, t)}^{-B(\tau, t)+A(\tau, t)} \exp \left(-\frac{(x-\eta)^{2}}{4 B(\tau, t)}\right) d \eta \\
& \quad * \sum_{k=0}^{\infty} \frac{1}{2^{k} k !}\left(\int_{\tau}^{t} \int_{\tau}^{t} b_{2}\left(s_{1}, s_{2}\right) d s_{1} d s_{2}\right)^{k} \frac{d^{4 k}}{d x^{4 k}} \mathrm{M} f(\tau, x) d \tau
\end{aligned}
$$

является математическим ожиданием (в обобщенном смысле) решения задачи (1), (2). 
ДоКАЗАТЕЛЬСТво. Воспользуемся формулой (16):

$$
\begin{gathered}
\mathrm{M} u(t, x)=\mathrm{M} u_{0}(x) \stackrel{x}{*} F_{\xi}^{-1}\left[\psi_{\varepsilon_{1}}\left(-\xi \chi\left(t_{0}, t, \cdot\right)\right)\right](x) \stackrel{x}{*} F_{\xi}^{-1}\left[\psi_{\varepsilon_{2}}\left(i \xi^{2} \chi\left(t_{0}, t, \cdot\right)\right)\right](x) \\
\quad+\int_{t_{0}}^{t} F_{\xi}^{-1}\left[\psi_{\varepsilon_{1}}(-\xi \chi(\tau, t, \cdot))\right](x) \stackrel{x}{*} F_{\xi}^{-1}\left[\psi_{\varepsilon_{2}}\left(i \xi^{2} \chi(\tau, t, \cdot)\right)\right](x) \stackrel{x}{*} \mathrm{M} f(\tau, x) d \tau
\end{gathered}
$$

Используя свойства преобразования Фурье [2], находим

$$
\begin{gathered}
F_{\xi}^{-1}\left[\psi_{\varepsilon_{1}}(-\xi \chi(\tau, t, \cdot))\right](x)=F_{\xi}^{-1}\left[\frac{\sin A(\tau, t) \xi}{A(\tau, t) \xi} \exp (-i \xi B(\tau, t))\right](x) \\
=\left\{\begin{array}{lll}
\frac{1}{2 A(\tau, t)} & \text { при }|x+B(\tau, t)|<A(\tau, t), \\
0 & \text { при }|x+B(\tau, t)|>A(\tau, t),
\end{array}\right. \\
F_{\xi}^{-1}\left[\psi_{\varepsilon_{2}}\left(i \xi^{2} \chi(\tau, t, \cdot)\right)\right](x)=\left(4 \pi \int_{\tau}^{t} \mathrm{M} \varepsilon_{2}(s) d s\right)^{-0.5} \exp \left(-\frac{x^{2}}{4 \int_{\tau}^{t} \mathrm{M} \varepsilon_{2}(s) d s}\right) \\
\quad * \sum_{k=0}^{\infty} \frac{1}{2^{k} k !}\left(\int_{\tau}^{t} \int_{\tau}^{t} b_{2}\left(s_{1}, s_{2}\right) d s_{1} d s_{2}\right)^{k} \frac{d^{4 k}}{d x^{4 k}} \delta(x) .
\end{gathered}
$$

Далее,

$$
\begin{gathered}
F_{\xi}^{-1}\left[\psi_{\varepsilon_{1}}(-\xi \chi(\tau, t, \cdot))\right](x) \stackrel{x}{*}\left(4 \pi \int_{\tau}^{t} \mathrm{M} \varepsilon_{2}(s) d s\right)^{-0.5} \exp \left(-\frac{x^{2}}{4 \int_{\tau}^{t} \mathrm{M} \varepsilon_{2}(s) d s}\right) \\
=\frac{1}{4 A(\tau, t)(\pi B(\tau, t))^{0.5}} \int_{-B(\tau, t)-A(\tau, t)}^{-B(\tau, t)+A(\tau, t)} \exp \left(-\frac{(x-\eta)^{2}}{4 B(\tau, t)}\right) d \eta, \\
\mathrm{M} u_{0}(x) * \sum_{k=0}^{\infty} \frac{1}{2^{k} k !}\left(\int_{\tau}^{t} \int_{\tau}^{t} b_{2}\left(s_{1}, s_{2}\right) d s_{1} d s_{2}\right)^{k} \frac{d^{4 k}}{d x^{4 k}} \delta(x) \\
=\sum_{k=0}^{\infty} \frac{1}{2^{k} k !}\left(\int_{\tau}^{t} \int_{\tau}^{t} b_{2}\left(s_{1}, s_{2}\right) d s_{1} d s_{2}\right)^{k} \frac{d^{4 k}}{d x^{4 k}} \mathrm{M} u_{0}(x) .
\end{gathered}
$$

Подставляя эти соотношения в выражение для $\mathrm{M} u(t, x)$, приходим к результату, приведенному в теореме. Теорема доказана.

9. Нахождение $Y_{2}$. Выпишем задачу (5), (6) для $Y_{2}$ :

$$
\begin{gathered}
\frac{\partial}{\partial t} Y_{2}\left(v_{1}, v_{2}, w, t, s_{2}, x, x_{2}\right)=-i \frac{\delta}{\delta v_{1}(t)} \frac{\partial}{\partial x} Y_{1}\left(v_{1}, v_{2}, w, t, s_{2}, x, x_{2}\right) \\
-i \frac{\delta}{\delta v_{2}(t)} \frac{\partial^{2}}{\partial x^{2}} Y_{1}\left(v_{1}, v_{2}, w, t, s_{2}, x, x_{2}\right)-i \frac{\delta}{\delta w(t, x)} Y_{1}\left(v_{1}, v_{2}, w, s_{2}, x_{2}\right) \\
Y_{2}\left(v_{1}, v_{2}, w, t_{0}, t_{0}, x, x_{2}\right)=\mathrm{M}\left(u_{0}(x) u_{0}\left(x_{2}\right)\right) \varphi\left(v_{1}, v_{2}, w\right) .
\end{gathered}
$$


Чтобы воспользоваться теоремой 1 для нахождения $Y_{2}\left(v_{1}, v_{2}, w, t, s_{2}, x, x_{2}\right)$, нужно знать начальное условие $Y_{2}\left(v_{1}, v_{2}, w, t_{0}, s_{2}, x, x_{2}\right)$, однако выполнено только условие (20). Оказывается, что условие симметричности $Y_{2}$ по переменным $(t, x),\left(s_{2}, x_{2}\right)$ позволяет найти $Y_{2}$.

Положим в уравнении (19) $s_{2}=t_{0}$. Получим задачу для $Y_{2}\left(v_{1}, v_{2}, w, t, t_{0}, x, x_{2}\right)$. Для нее применима теорема 1 , и мы находим

$$
\begin{aligned}
& Y_{2}\left(v_{1}, v_{2}, w, t_{0}, t, x_{2}, x\right)=Y_{2}\left(v_{1}, v_{2}, w, t, t_{0}, x, x_{2}\right) \\
& =F_{\xi}^{-1}\left[F_{x}\left[\mathrm{M}\left(u_{0}(x) u_{0}\left(x_{2}\right)\right) \varphi\left(v_{1}-\xi \chi\left(t_{0}, t, \cdot\right), v_{2}+i \xi^{2} \chi\left(t_{0}, t, \cdot\right), w\right)\right](\xi)\right](x) \\
& -i \int_{t_{0}}^{t} F_{\xi}^{-1}\left[F_{x}\left[\frac{\delta}{\delta w(\tau, x)} Y_{1}\left(v_{1}-\xi \chi(\tau, t, \cdot), v_{2}+i \xi^{2} \chi(\tau, t, \cdot), w, t_{0}, x_{2}\right)\right](\xi)\right](x) d \tau
\end{aligned}
$$

Отсюда получаем начальное условие для уравнения (19)

$$
\begin{gathered}
Y_{2}\left(v_{1}, v_{2}, w, t_{0}, s_{2}, x, x_{2}\right)= \\
=F_{\xi}^{-1}\left[F_{x}\left[\mathrm{M}\left(u_{0}\left(x_{2}\right) u_{0}(x)\right) \varphi\left(v_{1}-\xi \chi\left(t_{0}, s_{2}, \cdot\right), v_{2}+i \xi^{2} \chi\left(t_{0}, s_{2}, \cdot\right), w\right)\right](\xi)\right]\left(x_{2}\right) \\
-i \int_{t_{0}}^{s_{2}} F_{\xi}^{-1}\left[F _ { x _ { 2 } } \left[\frac { \delta } { \delta w ( \tau , x _ { 2 } ) } Y _ { 1 } \left(v_{1}-\xi \chi\left(\tau, s_{2}, \cdot\right),\right.\right.\right. \\
\left.\left.\left.v_{2}+i \xi^{2} \chi\left(\tau, s_{2}, \cdot\right), w, t_{0}, x\right)\right](\xi)\right]\left(x_{2}\right) d \tau .
\end{gathered}
$$

Снова воспользуемся теоремой 1 , получим

$$
\begin{gathered}
Y_{2}\left(v_{1}, v_{2}, w, t, s_{2}, x, x_{2}\right)=F_{\eta}^{-1}\left[F _ { x } \left[F _ { \xi } ^ { - 1 } \left[F _ { x _ { 2 } } \left[\mathrm { M } ( u _ { 0 } ( x _ { 2 } ) u _ { 0 } ( x ) ) \varphi \left(v_{1}-\xi \chi\left(t_{0}, s_{2}, \cdot\right)\right.\right.\right.\right.\right. \\
\left.\left.\left.-\eta \chi\left(t_{0}, t, \cdot\right), v_{2}+i \xi^{2} \chi\left(t_{0}, s_{2}, \cdot\right)+i \xi^{2} \chi\left(t_{0}, t, \cdot\right), w\right)\right](\xi)\right]\left(x_{2}\right) \\
-i \int_{t_{0}}^{s_{2}} F_{\xi}^{-1}\left[F _ { x _ { 2 } } \left[\frac { \delta } { \delta w ( \tau , x _ { 2 } ) } Y _ { 1 } \left(v_{1}-\xi \chi\left(\tau, s_{2}, \cdot\right)-\eta \chi\left(t_{0}, t, \cdot\right)\right.\right.\right. \\
\left.\left.\left.\left.\left.v_{2}+i \xi^{2} \chi\left(\tau, s_{2}, \cdot\right)+i \eta^{2} \chi\left(t_{0}, t, \cdot\right), w, t_{0}, x\right)\right](\xi)\right]\left(x_{2}\right) d \tau\right](\eta)\right](x) \\
-i \int_{t_{0}}^{t} F_{\xi}^{-1}\left[F _ { x } \left[\frac { \delta } { \delta w ( \tau , x ) } Y _ { 1 } \left(v_{1}-\xi \chi(\tau, t, \cdot)\right.\right.\right. \\
\left.\left.\left.v_{2}+i \xi^{2} \chi(\tau, t, \cdot), w, s_{2}, x_{2}\right)\right](\xi)\right](x) d \tau
\end{gathered}
$$

Мы доказали следующий результат.

Теорема 8. Если характеристический функционал $\varphi\left(v_{1}, v_{2}, w\right)$ имеетвариационнье производнье до третьего порядка включительно, $\mathrm{M} u_{0}(x)$ u $\mathrm{M}\left(u_{0}(x) u_{0}\left(x_{1}\right)\right)$ локально суммируемы, то (21) является единственным симметрическим по переменным $(t, x),\left(s_{2}, x_{2}\right)$ решением задачи (19), (20) в обобщенном смысле. 
10. Вторая моментная функция решения задачи (1), (2). Теперь из (21) при $v_{1}=0, v_{2}=0, w=0$ получаем вторую моментную функцию решения задачи (1), (2).

Теорема 9. Пусть выполняются условия теоремы 8. Тогда

$$
\begin{aligned}
\mathrm{M}(u(t, x) & \left.u\left(s_{2}, x_{2}\right)\right) \\
= & F_{\eta}^{-1}\left[F _ { x } \left[F _ { \xi } ^ { - 1 } \left[F _ { x _ { 2 } } \left[\mathrm{M}\left(u_{0}\left(x_{2}\right) u_{0}(x)\right)\right.\right.\right.\right. \\
& \left.\left.\times \varphi\left(-\xi \chi\left(t_{0}, s_{2}, \cdot\right)-\eta \chi\left(t_{0}, t, \cdot\right), i \xi^{2} \chi\left(t_{0}, s_{2}, \cdot\right)+i \xi^{2} \chi\left(t_{0}, t, \cdot\right), w\right)\right](\xi)\right]\left(x_{2}\right) \\
& -i \int_{t_{0}}^{s_{2}} F_{\xi}^{-1}\left[F _ { x _ { 2 } } \left[\frac { \delta } { \delta w ( \tau , x _ { 2 } ) } Y _ { 1 } \left(-\xi \chi\left(\tau, s_{2}, \cdot\right)-\eta \chi\left(t_{0}, t, \cdot\right),\right.\right.\right. \\
& \left.\left.\left.\left.\left.i \xi^{2} \chi\left(\tau, s_{2}, \cdot\right)+i \eta^{2} \chi\left(t_{0}, t, \cdot\right), w, t_{0}, x\right)\right](\xi)\right]\left(x_{2}\right) d \tau\right](\eta)\right](x) \\
- & i \int_{t_{0}}^{t} F_{\xi}^{-1}\left[F_{x}\left[\frac{\delta}{\delta w(\tau, x)} Y_{1}\left(-\xi \chi(\tau, t, \cdot), i \xi^{2} \chi(\tau, t, \cdot), w, s_{2}, x_{2}\right)\right](\xi)\right](x) d \tau
\end{aligned}
$$

является второй моментной функиией (в смысле обобщенных функиий) решения задачи (1), (2).

Отметим, что $Y_{1}$ определяется формулой (14). Кроме того, если в (22) положить $s_{2}=t, x_{2}=x$, то получим дисперсионную функцию решения задачи (1), (2).

11. Вторая смешанная функция. Из разложения характеристическогофункционала $Y$ в степенной ряд (см. п. 3) следует, что

$$
\begin{aligned}
Y_{1}\left(v_{1}, v_{2}, w, t, x\right)= & \mathrm{M}\left(u ( t , x ) \operatorname { e x p } \left(i \int _ { T } \left(\varepsilon_{1}(s) v_{1}(s)\right.\right.\right. \\
& \left.\left.\left.+\varepsilon_{2}(s) v_{1}(s)\right) d s+i \int_{T} \int_{\mathbb{R}} f\left(s_{1}, s_{2}\right) w\left(s_{1}, s_{2}\right) d s_{1} d s_{2}\right)\right) .
\end{aligned}
$$

Это позволяет находить смешанные моментные функции. Приведем один такой результат.

Теорема 10. Пусть случайнъе прочессы $\varepsilon_{1}, \varepsilon_{2}$ не зависят от прочесса $f$, $\psi\left(v_{1}, v_{2}\right)$ - характеристический функционал процессов $\varepsilon_{1}, \varepsilon_{2}-$ имеет вариационные производные до второго порядка включительно и $\mathrm{M} u_{0}(x), \operatorname{M} f(t, x)$ локально суммируемь. Тогда

$$
\begin{aligned}
\mathrm{M}\left(u(t, x) \varepsilon_{1}\left(t_{1}\right)\right)=\frac{1}{i} & \mathrm{M} u_{0}(x) \stackrel{x}{*} F_{\xi}^{-1}\left[\frac{\delta \psi\left(-\xi \chi\left(t_{0}, t, \cdot\right), i \xi^{2} \chi\left(t_{0}, t, \cdot\right)\right)}{\delta v_{1}\left(t_{1}\right)}\right](x) \\
& -i \int_{t_{0}}^{t} F_{\xi}^{-1}\left[\frac{\delta \psi\left(-\xi \chi(\tau, t, \cdot), i \xi^{2} \chi(\tau, t, \cdot)\right)}{\delta v_{1}\left(t_{1}\right)}\right](x) \stackrel{x}{*} \mathrm{M} f(\tau, x) d \tau
\end{aligned}
$$

является второй моментной функцией $\mathrm{M}\left(u(t, x) \varepsilon_{1}\left(t_{1}\right)\right)$ (в смисле обобщенных функиий) для решения задачи (1), (2). 
ДокАЗАТЕльство. Воспользуемся найденным выражением (14) для $Y_{1}$. При этом

$$
\begin{aligned}
& \mathrm{M}\left(u(t, x) \varepsilon_{1}\left(t_{1}\right)\right)=\left.\frac{1}{i} \frac{\delta Y_{1}\left(v_{1}, v_{2}, w, t, x\right)}{\delta v_{1}\left(t_{1}\right)}\right|_{v_{1}=0, v_{2}=0, w=0} \\
& =\left\{\frac{1}{i} \mathrm{M} u_{0}(x) \stackrel{x}{*} F_{\xi}^{-1}\left[\frac{\delta \varphi\left(v_{1}-\xi \chi\left(t_{0}, t, \cdot\right), v_{2}+i \xi^{2} \chi\left(t_{0}, t, \cdot\right), w\right)}{\delta v_{1}\left(t_{1}\right)}\right](x)\right. \\
& \left.-\int_{t_{0}}^{t} F_{\xi}^{-1}\left[F_{x}\left[\frac{\delta^{2} \varphi\left(v_{1}-\xi \chi(\tau, t, \cdot), v_{2}+i \xi^{2} \chi(\tau, t, \cdot), w\right)}{\delta w(\tau, x) \delta v_{1}\left(t_{1}\right)}\right](\xi)\right](x) d \tau\right\}\left.\right|_{\begin{array}{l}
v_{1}=0 \\
v_{2}=0 \\
w=0
\end{array}} \\
& =\frac{1}{i} \mathrm{M} u_{0}(x) \stackrel{x}{*} F_{\xi}^{-1}\left[\frac{\delta \psi\left(-\xi \chi\left(t_{0}, t, \cdot\right), i \xi^{2} \chi\left(t_{0}, t, \cdot\right)\right)}{\delta v_{1}\left(t_{1}\right)}\right](x) \\
& -i \int_{t_{0}}^{t} F_{\xi}^{-1}\left[F_{x}\left[\frac{\delta \psi\left(-\xi \chi(\tau, t, \cdot), i \xi^{2} \chi(\tau, t, \cdot)\right)}{\delta v_{1}\left(t_{1}\right)} \mathrm{Mf}(\tau, x)\right](\xi)\right](x) d \tau \\
& =\frac{1}{i} \mathrm{M} u_{0}(x) \stackrel{x}{*} F_{\xi}^{-1}\left[\frac{\delta \psi\left(-\xi \chi\left(t_{0}, t, \cdot\right), i \xi^{2} \chi\left(t_{0}, t, \cdot\right)\right)}{\delta v_{1}\left(t_{1}\right)}\right](x) \\
& -i \int_{t_{0}}^{t} F_{\xi}^{-1}\left[\frac{\delta \psi\left(-\xi \chi(\tau, t, \cdot), i \xi^{2} \chi(\tau, t, \cdot)\right)}{\delta v_{1}\left(t_{1}\right)} F_{x}[\mathrm{M} f(\tau, x)](\xi)\right](x) d \tau .
\end{aligned}
$$

Отсюда следует искомый результат. Теорема доказана.

12. Заключение. Полученные формулы для математического ожидания (15) и для второй моментной функции (22) являются довольно общими. Они могут быть использованы для уравнений, коэффициенты которых являются различными случайными процессами. Рассмотренньй пример с гауссовскими случайными коэффициентами показывает, что для конкретных процессов формулы допускают дальнейшие упрощения. Формулы (14) для $Y_{1}$ и (21) для $Y_{2}$ позволяют находить некоторые смешанные моментные функции более высокого порядка с помошью вычисления вариационных производных (см. п. 11). Из вида формулы (21) для $Y_{2}$ трудно усмотреть ее симметричность по переменным $(t, x)$ и $\left(s_{2}, x_{2}\right)$. Для этого нужно подставить в нее найденное ранее значение $Y_{1}$.

Автор благодарен профессору Н.Х. Розову и профессору В. М. Миллионшикову за полезные обсуждения проблемы.

\section{Список литературы}

1. Данфорд Н., Швари Д. Линейные операторы. Т. І. М.: ИЛ, 1962.

2. Владимиров B. C. Обобщенные функции в математической физике. М.: Наука, 1976.

3. Колмогоров А. Н., Фомин С. В. Элементы теории функций и функционального анализа. М.: Наука, 1976.

4. Задорожний В. Г. Дифференциальные уравнения с вариационными производными. Воронеж: ВорГУ, 2000.

5. Кляцкин В.И. Стохастические уравнения и волны в случайно-неоднородных средах. М.: Наука, 1980. 
6. Фурсиков $A$. В. Проблема замыкания цепочек моментных уравнений, соответствующих трехмерной системе уравнений Навье-Стокса в случае больших чисел Рейнольдса // ДАН СССР. 1991. Т. 319. № 1. С. 83-87.

7. Фурсиков A. B. Моментная теория для уравнений Навье-Стокса со случайной правой частью // Изв. РАН. Сер. матем. 1992. Т. 56. №6. С. 1273-1315.

8. ШІапиро В.Е., Логинов В. М. Динамические системы при случайных воздействиях. Новосибирск: Наука, 1983.

9. Тихонов В. И. Воздействие флуктуаций на простейшие параметрические системы // Автоматика и телемеханика. 1958. Т. 19. № 8. С. 717-723.

10. Адомиан Дж. Стохастические системы. М.: Мир, 1987.

11. Вентцель А. Д., Фрейдлин М. И. Флуктуации в динамических системах под действием малых случайных возмущений. М.: Наука, 1979.

12. Монин А.С., Яълом А.М. Статистическая гидромеханика. Ч. 2. М.: Наука, 1967.

13. Задорожний В.Г., Строева Л.Н. О моментных функциях решения начальной задачи линейного дифференциального уравнения первого порядка со случайными коэффициентами // Дифференц. уравн. 2000. Т. 36. № 3. С. 377-385.

14. Задорохний В.Г. Дифференциальное уравнение в банаховом пространстве, содержащее вариационную производную // СМЖ. 1992. Т. 33. № 2. С. 80-93.

15. Задорожний В. Г. Моментные функции решения задачи Коши стохастического уравнения теплопроводности // Докл. РАН. 1999. Т. 364. №6. С. 735-737.

16. Шилов Г. Е. Математический анализ. Второй специальный курс. М.: Наука, 1965.

Воронежский государственный университет

Поступило в редакцию

E-mail: zador@zador.vsu.ru

09.I.2001 